Supplementary Information for

\title{
Achieving Infrared Detection by All-Si Plasmonic Hot-Electron Detectors with High Detectivity
}

Bo Feng, Jingyuan Zhu, Bingrui Lu, Feifei Liu, Lei Zhou and Yifang Chen*

$\S$ Nanolithography and Application Research Group, State Key Lab of Asic and System,

School of Information Science and Technology, Fudan University, Shanghai, 200433, China.

IDepartment of Physics, Fudan University, Shanghai, 200433, China.

Corresponding authors: yifangchen@fudan.edu.cn (Yifang Chen)
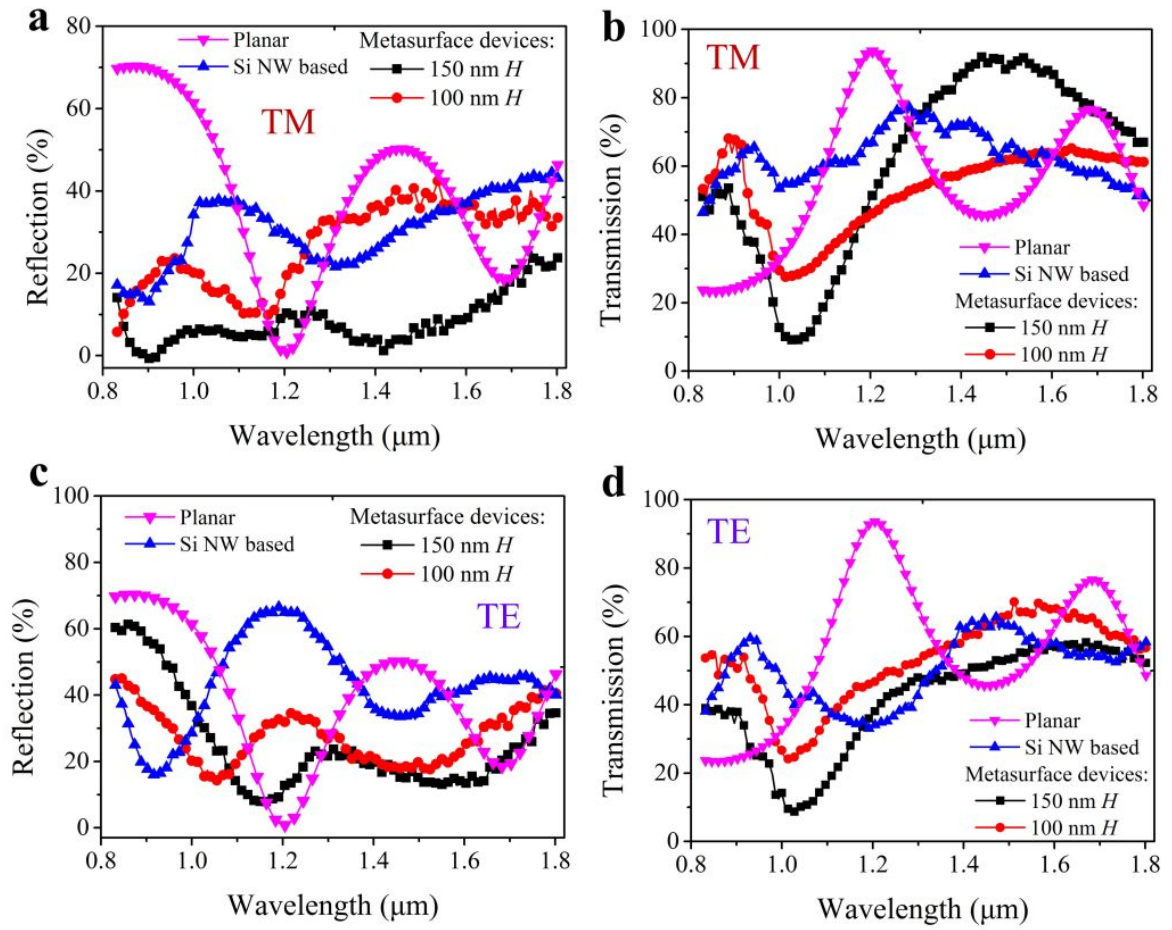
Figure S1. The measured optical reflection, transmission spectra of the light with TM and TE polarization, respectively, for the all-Si based detectors with the four configurations. While the device was illuminated by TM-polarized or TE-polarized light at 1.0-1.8 $\mu \mathrm{m}$ wavelength, the transmissions through the device without antennas $(\mathrm{b}, \mathrm{d})$ is mostly above $50 \%$, corresponding to low absorption by planar $\mathrm{Si}$ as expected. Adding the designed antenna to the device, both the reflection and the transmission of TM polarized $(\mathrm{a}, \mathrm{b})$ as well as TE polarized light $(\mathrm{c}, \mathrm{d})$ remain low, resulting in significant absorption peaks in $1.0-1.8 \mu \mathrm{m}$.

Table S1 | Comparisons of the device performances between our detector and the ones based on hot carriers from literatures, PV, Photovoltaic; PC, photoconductive.

\begin{tabular}{lcccccc}
\hline $\begin{array}{l}\text { All-Si device } \\
\text { structure }\end{array}$ & Type & $\begin{array}{c}\text { Operating } \\
\text { voltage }\end{array}$ & $\begin{array}{c}\text { Peak responsivity } \\
(\mathrm{mA} / \mathrm{W})\end{array}$ & $\begin{array}{c}\text { Quantum } \\
\text { efficiency }\end{array}$ & $\begin{array}{c}\text { Peak detectivity } \\
\text { ( Jone) }\end{array}$ & Refs \\
\hline This work & PC & $1.5 \mathrm{~V}$ & $\begin{array}{c}94.5 @ \lambda=1.15 \mu \mathrm{m} \\
18.2 @ \lambda=1.55 \mu \mathrm{m}\end{array}$ & $\begin{array}{c}11.7 \% \\
1.5 \%\end{array}$ & $\begin{array}{c}4.38 \times 10^{11} \\
8.4 \times 10^{10}\end{array}$ & \\
Metal Grating & PV & $0 \mathrm{~V}$ & $0.6 @ \lambda=1.45 \mu \mathrm{m}$ & $\sim 0.2 \%$ & $/$ & {$[7]$} \\
$\begin{array}{l}\text { DTTM } \\
\text { antenna }\end{array}$ & PV & $0 \mathrm{~V}$ & $4.1 @ \lambda=1.44 \mu \mathrm{m}$ & $/$ & $/$ & {$[13]$} \\
$\begin{array}{l}\text { Pyramid } \\
\text { Si/Au }\end{array}$ & PV & $0 \mathrm{~V}$ & $8.17 @ \lambda=1.2 \mu \mathrm{m}$ & $\sim 0.8 \%$ & $1.78 \times 10^{10}$ & {$[14]$} \\
MPA & PV & $0 \mathrm{~V}$ & $1.25 @ \lambda=1.25 \mu \mathrm{m}$ & $/$ & & {$[15]$} \\
Metamaterials & PV & $0 \mathrm{~V}$ & $2.2 @ \lambda=1.33 \mu \mathrm{m}$ & $0.2 \%$ & & / \\
& $-1.5 \mathrm{~V}$ & $3.7 @ \lambda=1.33 \mu \mathrm{m}$ & $\sim 0.34 \%$ & & {$[17]$} \\
\hline
\end{tabular}

\section{Lorentzian line shape for the theoretical plasmon absorption spectrum $S(v)$}

In order fit the experimental date for responsivity and EQE, we carried out a series of Lorentzian line shape theory. In general, the theoretical plasmon absorption spectrum $S(v)$ depends on multiple factors, including the composition, geometry, and size of the devices. In the 
quasi-static system, the optical response exhibits a Lorentzian line shape near the resonance frequency. ${ }^{1,2}$ Based this, the theoretical plasmon absorption, satisfied a Lorentzian line shape function is calculated as follow:

$$
S(v)=\frac{w}{4 \pi^{2}} \cdot \frac{1}{\left(v-v_{0}\right)^{2}+\left(\frac{w}{2}\right)^{2}}, \text { where } w \text { is the line width. }
$$

To clearly illustrate this concept, the schematic diagram is plotted, as follow:

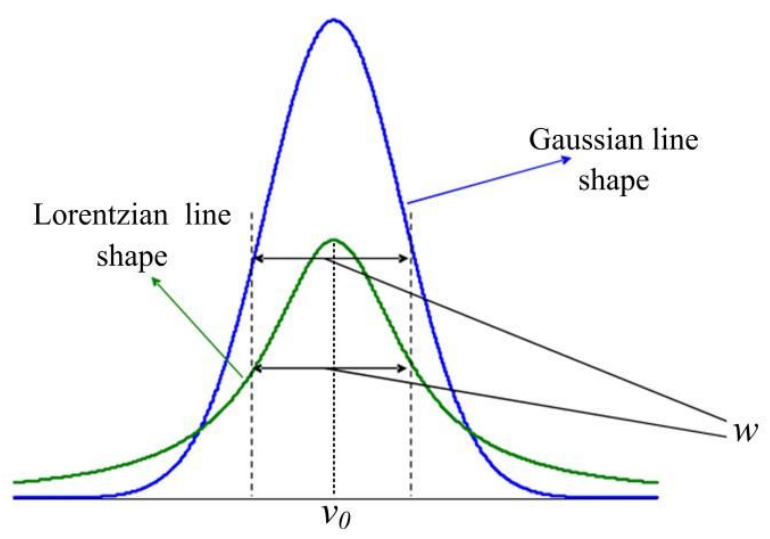

Figure S2. The schematic diagram of the Lorentzian line shape and Gaussian line shape.

Based this, by fitting the measured responsivity (points) and $E Q E$ (points) of the metasurface devices with Eq. (1), and Eq. (2), respectively, the $w$ and $v_{0}$ are also extracted as $6.247 \times 10^{14} \mathrm{~Hz}$ ( $\sim 480 \mathrm{~nm}$ band width), and $2.61 \times 10^{14} \mathrm{~Hz}(\sim 1.15 \mu \mathrm{m}$ wavelength), respectively, for the wire height of $150 \mathrm{~nm}$ at TM-polarized light illumination. 


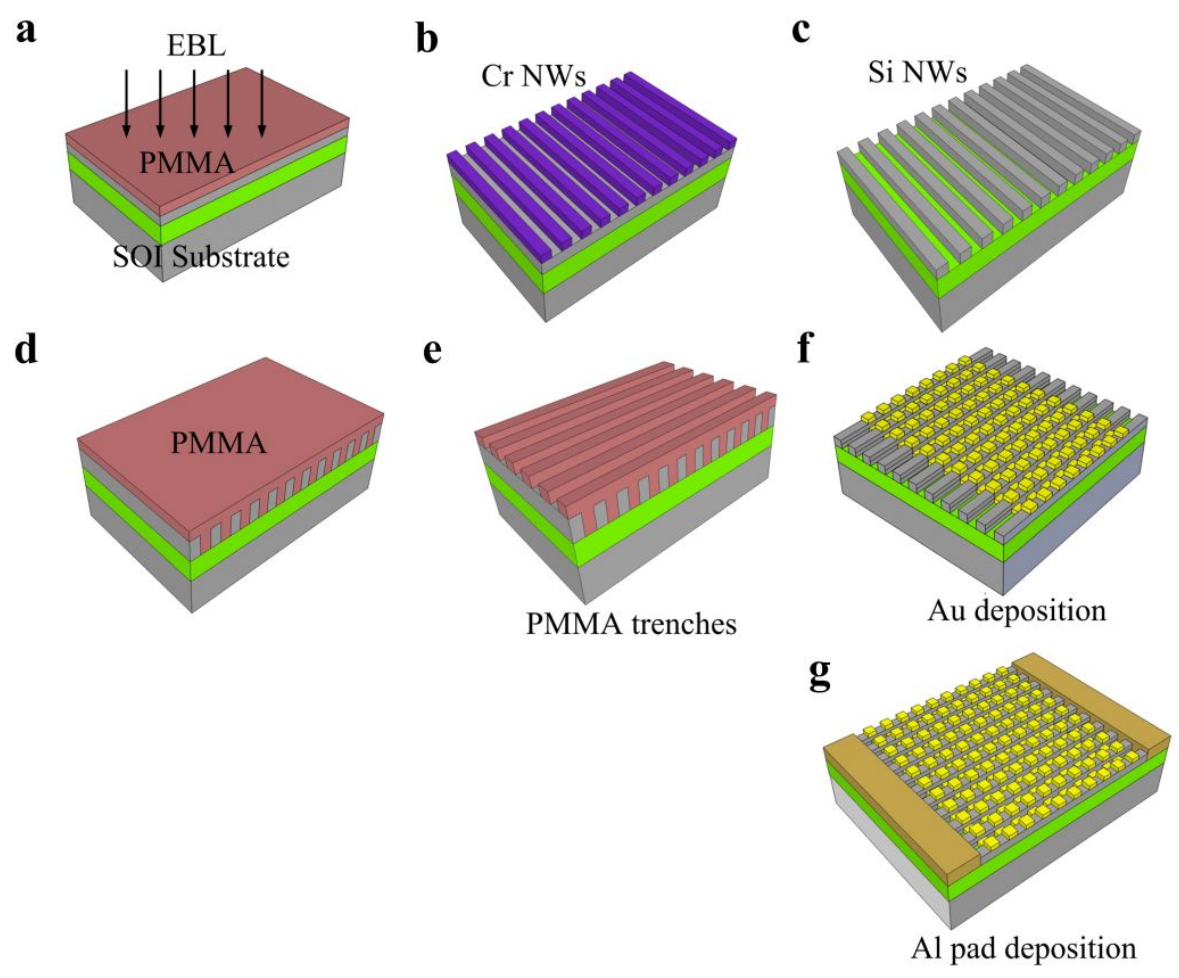

Figure S3. The process flow in the nanofabrication of the all Si-based detector. (a) E-beam exposure on the PMMA coated on the top of the Si on insulator (SOI) substrate. (b) After development and metallization by $\mathrm{Cr}$ and lift off, $\mathrm{Cr}$ nanolines are replicated on the top. (c) Reactive ion etch (RIE) using the pre-patterned $\mathrm{Cr}$ as etch mask to form nanowires in Si. The Cr-mask was removed by a Chromium etchant solution. (d) Coating of a PMMA layer for the second electron beam lithography. (e) After EBL, trenches in PMMA perpendicular to the Si nanowires are formed. (f) Deposition of 2-nm Ti/40-nm Au bi-layer followed by a lift off forms the golden antennas. Annealing in nitrogen gas at $500{ }^{\circ} \mathrm{C}$ for $15 \mathrm{~min}$ was undertaken, aiming to reduce the surface defects on the wires. (g) The two terminal contacts are formed by optical lithography, followed by Al metallization to from electric measurement pads.

\section{References:}

1. Knight, M. W.; Sobhani, H.; Nordlander, P.; Halas, N. J. Photodetection with Active Optical Antennas. Science. 2011, 332, 702-704.

2. Kreibig U.; Vollmer M. Optical Properties of Metal Clusters. Springer, New York, 1995. 